\title{
Using Astronomy as a Vehicle for Science Education
}

\author{
Case Rijsdijk \\ South African Astronomical Observatory, \\ PO Box 9, Observatory, 7935, South Africa \\ case@saao.ac.za \\ Received 1999 August 19, accepted 2000 April 7
}

\begin{abstract}
Recent history in South Africa has created unique problems in many areas. As a result of an unequal educational structure, particularly in science, there is a general lack of science literacy and awareness. Some years ago the South African Astronomical Observatory started a Science Education Initiative as a contribution to addressing this problem by using astronomy as a vehicle to stimulate an interest in science amongst teachers and students. This programme was given a boost by the South African government in 1998 and the construction of the Southern African Large Telescope will provide further stimulus to the Science Education Initiative.
\end{abstract}

Keywords: astronomy education

\section{The Science Education Initiative}

The South African Astronomical Observatory (SAAO) began an education programme, in addition to its lowprofile public awareness programme, on a part-time basis in 1994. In line with recent government policy on Science and Technology, this became an integral part of the SAAO in 1996 with the appointment of a full-time education officer and the development of the Science Education Initiative (SEI). Traditionally, astronomy was taught in South African schools as an introduction to some aspects of the geography syllabus. The astronomy content was minimal and often badly taught or omitted, depending on the knowledge and ability of the teacher. Since basic scientific research was often seen as an elitist luxury, and the fact that much science education took place within the ambit of environmental education, meant that the following three statements were particularly relevant to the development of the SEI:

Scientific endeavour is not purely utilitarian in its objectives and has important associated cultural and social values. It is also important to maintain basic competence in flagship sciences such as physics and astronomy for cultural reasons. Not to offer them would be to take a negative view of our future- the view that we are a second-class nation chained forever to the treadmill of feeding and clothing ourselves (Department of Arts, Culture, Science and Technology 1996).

\begin{abstract}
A knowledge of astronomy produces a humility that creates a respect for planet Earth and an awareness of the delicacy of its environment (Rijsdijk 1995).

One of the problems of our time is to overcome the attitudes that tend to justify and reinforce the isolation of the scientific community. We must open new channels of communication between science and society (Prigogine 1980).
\end{abstract}

The SEI set out some specific goals (Rijsdijk 1996) that can be summarised briefly as:

- using astronomy to stimulate young people to follow careers in Science, Engineering and Technology (SET) through a student and teacher science education programme

- developing resources for students and teachers

- encouraging students and teachers to attend workshops at both the SAAO and their own schools

- re-introducing astronomy in the national schools' curriculum

- using astronomy to stimulate an interest in science

- raising public awareness of astronomy and the SAAO.

The underlying philosophy was to use astronomy as a vehicle to stimulate an interest in science, rather than to actually teach astronomy. Of course when and where possible astronomy would be taught!

\section{Implementing the Science Education Initiative}

\subsection{Resources Centre}

In order to achieve some of the above objectives, a Science Education Resources Centre was set up inside an old disused storeroom at the Observatory in Cape Town. This was refurbished and deliberately left simple and 'low-tech'. The reasoning was to show teachers and students that much can be achieved with minimum facilities and that such a centre could be built almost anywhere. It is large enough to accommodate a group of about 25 people at a time, an ideal size for one demonstrator/facilitator to cope with.

The centre is fitted out with flat, unobstructed working surfaces and equipped with a pull-down screen, a few power points, an overhead projector and a storeroom/storage area. This allows a wide range of demonstrations to be done in addition to providing space for workshops.

Equipment used for demonstrations is usually made up from scraps and other odds and ends, such as empty plastic soft drink bottles, styrofoam cups, curtain rods, etc. Other equipment is made by students who undergo 
training in the SAAO workshops. Items such as power supplies, electronic timers, simple photometers etc. broaden the capability of the centre.

Additional equipment consists of small portable telescopes from $110 \mathrm{~mm}$ to $250 \mathrm{~mm}$ in diameter. All these have solar filters to allow direct viewing of the Sun. Slide, video and data projectors are also available to supplement and enrich longer workshops. In order to explain how modern astronomers collect data, a CCD camera is attached to the $250-\mathrm{mm}$ telescope and the image projected with the data projector using a laptop computer.

\subsection{Resource Development}

A transformation in the educational structures in South Africa has led to the government adopting an Outcomes Based Education (OBE) system known as Curriculum 2005. The 'Natural Sciences' is one of eight learning areas in the new curriculum and covers all sciences. The learning area itself is broken down into four 'themes', one of which 'Earth and Beyond' is subdivided into four sub-themes: 'Under the Earth's Surface', 'On the Earth's Surface', 'Above the Earth's Surface' and 'Beyond the Earth's Surface'. The latter contains a great deal of astronomy (Department of Education 1997). The government has started implementing Curriculum 2005 and this has influenced the type of resources that the SEI is developing. The implementation process is not easy when the majority of schools in South Africa, especially those in previously disadvantaged areas, are beset with problems such as:

- redeployment and rationalisation of teachers'right sizing'

- under-trained and under-qualified teachers

- large classes (45-60)

- poor facilities

- a lack of resources

- the lack of a 'culture of science'

- the lack of a 'culture of learning'.

With issues such as these it was clear that whatever resources were developed by the SEI, they needed to:

- be simple

- use cheap and readily available materials/scrap

- develop modules (with 'manuals') and workshop these modules with teachers

- be easily and cheaply reproducable enabling them to be taken straight into the classroom

- focus on skills development and group work

- be supported with assessment material and activity sheets.

To date about 20 of these modules have been developed, as well as a large number of activities and assessment materials. These modules are normally tried out on teachers or students before making them gen- erally available to teachers. Many of these modules use the making of simple models to illustrate the principles involved. At the end of each module is a set of full size drawings that can be photocopied onto card so that each student will be able to make his/her own model. Some typical examples include modules on:

- a simple telescope, Galilean and astronomical

- the Sunometer-a device explaining the movement of the Sun across the sky

- the Luxometer-a simple device to show the relation between light intensity and angle of incidence

- a simple planisphere

- a quadrant

- some Moon phase models and exercises

- several simple sundials

- how to make a spectroscope.

Because astronomy now forms an integral part of the new curriculum, many teachers sought help in preparing material for lessons on astronomy and related subjects. A number asked for specific material on topics such as eclipses, seasons, planets and the Moon and its phases. Most of these have been reworked into modules and the SEI is encouraging teachers to come forward with further ideas.

Some modules are sequential. For example, there is a series that shows how to verify the inverse-square law, which is followed by another on calculating the power of the Sun, which then enables students to find the distance to a star using a third module.

Because Curriculum 2005 emphasises an integrated approach, many of these resources are designed to give easy access to cross-curricular activities. An example of this is the 'Seascope', a device that can be used to look under-water for environmental studies. Other modules contain material from other learning areas: for example, some African starlore stories were transformed into a playlet and acted out by some primary school children. Such an activity combined astronomy, drama, music, arts and crafts (Stone 1998).

\subsection{Workshop Experiences}

When the SEI started, the principal target group was secondary (high) school teachers. It has however become quite clear that this group is far from ideal, the two main reasons being:

- much of the material covered by the SEI is not in the current syllabus

- most teachers see no reason to 'waste' time on this: both teachers and students are totally committed to preparing for their matriculation exams-rote learning at its worst!

For this reason, and the fact that Grade 9 is now seen as an exit point for students, the target group is now teachers and students from Grades 6-9. These students 
are typically aged $10-15$ years old, although it is possible to have much older students in these classes. Another reason for targeting this group is that this is where the new curriculum is being implemented first. This also ties up very well with the Teacher Training Colleges and several non-government education organisations such as the Primary Science Project. The SEI has built up a good working relation with such groups as they are ideal vehicles for workshopping and testing new materials.

Experience over the last two years has shown that children are curious and definitely like doing things such as:

- launching rockets

- making telescopes

- making quadrants

- making and using planispheres.

The well known problem that 'stars come out at night—students don't' is as true in South Africa as it is in other parts of the world-although for different reasons. In most parts of the world this is due to a lack of enthusiasm. In South Africa it is related to an inability to travel to the SAAO for various reasons, including a lack of vehicles, safety, costs and distance to be travelled.

The education techniques used have been 'Minds on, Hands on', getting students to become active roleplayers in their education, in line with the OBE approach. This what the SEI calls the CCD approach. Often a well chosen, simple demonstration creates a 'WOW' amongst students and this WOW factor creates a Curiosity, that can be related to the Curriculum and then Developed into a complete educational unit.

The procedure in running workshops has become quite routine. Usually materials or kits are handed out, then checked to make sure that everyone has the right materials. It is quite common for participants in workshops not to complete their project because they were missing something and are reluctant to ask questions. Some examples of typical workshops are:

Making telescopes: Halfway through the workshop the function and use of lenses is explained. This allows time for glue to set, after which the telescope is completed.

Newton's Laws: Many demonstrations explaining the effects of free fall are performed. Often students also make quadrants to measure heights and they then use these to measure how high water rockets go. This is a very popular cross-curricular activity as it involves the Learning Areas of Natural Science, Mathematics and Technology.

Our place in the Universe: Games and simulations with inflatable Earth globes and balls are used to model and explain the Moon's phases, the scale of the Solar System and astronomical objects. Where possible this is supplemented with a slide show or video such as 'Powers of Ten' (Eames \& Eames 1989).

Seasons: Models such as the Luxometer to measure changing light intensity and a Sunometer to measure changes in the Sun's altitude and length of day throughout the year, showing how these factors influence the seasons. Should time allow, a solar oven is also made.

The aim of these workshops is not only to help both teachers and students get a better understanding of astronomy and science, but also to try and establish an awareness of the environment on the largest possible scale. These workshops should be seen as an integral part of teaching and not merely as an interesting interlude in the routine of the day. They are designed to encourage observational skills and the ability to relate these observations to daily experience, thus engendering a sense of wonder and curiosity. In addition it is hoped that these workshops give teachers and students a basic understanding of astronomy and the science and technology that supports it.

\section{Friends with the Universe}

In 1997 the Department of Arts, Culture, Science and Technology announced that 1998 would be the Year of Science and Technology (YEAST) in South Africa. As a part of YEAST each of the eight science councils were awarded a sum of money for their 'thrust' within YEAST. The Foundation for Research Development chose as its thrust 'Astronomy and Space Science', targeting three of the nine provinces in South Africa, namely the Western Cape, Northern Cape and Gauteng. The SAAO submitted a business plan to the Department on behalf of the Foundation (Rijsdijk 1998) entitled 'Friends with the Universe'. This project had six broad aims:

- extend the present workshops held at the SAAO and also hold one major national workshop as part of the IAU Symposium 192

- invite prominent local and overseas, speakers to address the South African public

- subsidise transport for people from the disadvantaged community enabling them to attend these talks

- produce a series of nine posters to be distributed nationally

- upgrade facilities at local planetaria and observatories to improve their capability of raising public awareness

- create a mobile facility to take science to the rural communities in the Western Cape, Northern Cape and Gauteng.

The last of these developed into the 'Starbus' project (see below). The 'Friends with the Universe' was most successful, the highlights being: 
- The visits by Dr David Malin, Professors John Barrow, Julietta Fierro and Gibor Basri

- The nine posters produced, which proved to be extremely popular, especially the first one on African Starlore. They were designed with the dual purpose of being both decorative and educational, and were distributed nationally

- The 'Astronomy in Education' workshop held in Cape Town after the IAU 192 Symposium (IAU Commission 1999).

\section{The 'Starbus' Project}

The 'Starbus' is in fact a colourfully decorated mini-bus equipped with a great deal of equipment, both high-tech and the developed low-tech resource materials of the SEI. This combination enables SAAO staff to take the successful SEI workshops to more remote areas. During such workshops students, teachers and members of the local community are exposed to wide variety of materials and equipment, ranging from shadow sticks to computer-driven telescopes. It was in fact a collaborative effort between Department, the Foundation and the SAAO, since the SAAO bought the vehicle, developed the resources and supplied the staff to run it with the equipment being funded by all three organisations. The success of the 'Starbus' during YEAST was such that it now forms an integral part of the SEI.

\subsection{Using the Starbus}

Generally schools that the 'Starbus' visits are exDepartment of Education and Training (DET) high schools in rural or peri-urban areas. These schools were run by the previous government for blacks only and now form the core of what are commonly referred to as 'disadvantaged' schools. When visiting rural ex-DET schools the 'Starbus' would be on the road for about four days and in that time visit between six and eight such schools. In between these trips, there are frequent excursions to local ex-DET schools and often there are additional trips to other local groups such as school ecological societies, science clubs, Scouts and Girl Guides, etc.

Planning a trip takes time and, whilst not difficult, can be frustrating in that communicating with schools can be difficult. Often there is only one telephone, but the process followed now is:

- contact is established with the Head of Science or the Principal of the school

- the aim of the visit is explained

- the class or classes to be used are identified

- the programme is discussed-here teachers select from a number of different workshop modules, decide which ones they want, and then the SAAO staff put together a 'package' lasting about two hours

- the time, date and facilities available are established
- this discussion is then confirmed by fax, where possible

- the visit is confirmed a day or two before departure of the 'Starbus'.

Most workshops are held with groups of 40 students. Teachers select which classes or individual students can attend-usually these are Science and/or Geography students in Grades 6-10. The 'Starbus' is then prepared with the appropriate resources and materials for that particular visit. Usually several extra items are included so that the programme can be made as flexible as possible.

While the workshops are suitable for groups of 40 students, their impact is maximised by doing additional activities for larger groups in the playground during tea and lunch breaks. This includes launching water rockets and setting up the $114 \mathrm{~mm}$ and $200 \mathrm{~mm}$ telescopes to show solar images and sunspots. During inclement weather, such activities are replaced with slide shows or illustrated talks using OHP viewgraphs. The latter are easier to use since often there are no facilities to darken rooms during the day.

Experience with the 'Starbus' has shown that schools are, without exception, keen to be visited, once the objectives of the programme have been explained to staff-despite the fact that visits cause a certain amount of disruption.

Marketing skills have been refined-most teachers are intimidated by the word 'astronomy', on the other hand the phrase 'we're running science and technology workshops' holds a lot of appeal! (Heese 1998)

The 'Starbus' is also used to take equipment to Science Festivals and other major science events. The SASOL SciFest (SASOL is the registered trade name of the sponsoring company) has over the last three years become South Africa's major science festival, attracting over 40,000 people. The SAAO has been a major contributor to SciFest by running many of the SEI workshops, which have always been oversubscribed. Portable display boards, posters and models of major telescopes are also transported and displayed to promote awareness of astronomy and the SAAO.

\subsection{Problems Encountered}

The 'Starbus' project has only been operational for about 18 months and those involved with the project are all on a steep learning curve. Many of the problems that have been encountered were not anticipated and this often led to makeshift changes to plans. Some of these problems were:

- Teachers are overworked and very seldom able to attend the workshops, leaving SAAO staff in control of the group. Besides the discipline problems encountered, the absence of teachers means that the work covered during the workshop is seldom reinforced by teachers at school and visits 
are seen as isolated activities not related to school/ classwork

- Schools often lack the most simple resources (electricity, spare classrooms etc.) to accommodate some of the workshops

- The lack of security, especially in the urban and peri-urban areas, makes it difficult to visit schools, clubs or societies at night and so students lose that concept of 'looking up' and the opportunity to learn about the night sky

- Most teachers see astronomy as a highly specialised field and lack the confidence to encourage an interest in astronomy amongst students or to set relevant assignments. They are reluctant to participate in workshops and run follow-up activities. The 'Starbus' visit is often seen as a visit by 'experts' dealing with a topic outside of and beyond the curriculum.

It is impossible to anticipate what to expect on arriving at a school. This means that a quick assessment needs to be undertaken and the appropriate adjustments made. The programme thus needs to be extremely flexible and able to adapt to local conditions quickly.

Students' knowledge of astronomy is extremely limited and dependent on their immediate environment. In trying to make sense of what they see and know, students usually contrive complicated theories that have to be corrected before they can begin to understand basic astronomy. The video 'A Private Universe' (Project STAR 1989) clearly illustrates this point. Students might often have a fairly good understanding of the physics, yet struggle to apply this in any way to what they know or see. Their misconceptions are often complex and deep rooted (Enderstein \& Spargo 1998).

Many schools visited have rigid, conservative approaches to education and questioning is not encouraged. Staff running the 'Starbus' are encouraged to take a more relaxed approach. Once the ice is broken and students see that they aren't being tested in any way, their questions are uncontrived and wide ranging. In a typical session, questions dealing with the whereabouts of heaven to the salaries of astronomers could be encountered!

Another general problem that has been experienced is that students have little concept of the large numbers involving size, distances or time relating to astronomy. For example, many believe, that the Earth (and the Universe) began 2000 years ago (it is after all the beginning of the third millennium!), that stars are fairly accessible and that the Sun is smaller than the Moon. On other occasions trying to convince participants in an adult education programme that the Earth was not flat proved difficult. These again are reasons for keeping workshops as flexible and open-ended as possible with additional resources readily available. Students often contribute to the direction they take.

\subsection{Summary}

It is difficult to assess the impact of these visits other than by the response of the students and teachers. All schools, without exception, have asked for return visits. One of the greatest strengths of the 'Starbus' is that it is equipped to meet local requirements; schools only have to provide students, a classroom and the time. Anyone who has experienced the logistics of taking children on outings will appreciate the benefits of such a mobile facility (Heese 1998).

A great strength of the SEI and the 'Starbus' programme is that it gives students the space and opportunity to ask questions, wonder why and explore possibilities. In a school system which has tended to overemphasise factual knowledge and content, astronomy encourages a different approach to science and technology.

Visiting schools with the 'Starbus' has been shown to be an effective way of overcoming many of the problems in reaching students in the rural areas. The workshops not only teach students about the science and technology behind astronomy, but also encourages them to observe the world around them and so develop a curiosity about their environment in general, and science in particular. In addition it gives them some of the knowledge and skills required to understand their place in the Universe.

I have extremely powerful memories of excited children looking at the Moon in the clear Karoo sky through the $200 \mathrm{~mm}$ telescope, being able to name stars for the first time, and identify the constellations. Such experiences leave one with little doubt about the value of the project (Heese 1998).

\section{The Collateral Benefits of SALT}

The SAAO is about to embark on the construction of the Southern African Large Telescope (SALT). This is a major international collaboration for the construction of a 9 m class spectroscopic survey telescope, a twin of the Hobby-Eberly Telescope at McDonald Observatory, Texas. Such a major scientific development on the subcontinent will be used as a focal point to promote science and technology in the region.

One of the ways this will be accomplished is through the construction of a modern Visitor Centre (or 'Stargate') in close proximity to the large telescope. The telescope dome will incorporate a viewing gallery that will allow visitors to experience the full scale of SALT. Since spectroscopy forms the cornerstone of research done by SALT, a display called 'Fingerprinting Light' will be incorporated into the centre.

The South African government is funding half of the cost of SALT and as part of the entire project a Collateral Benefits Plan has been drawn up which identifies the benefits of SALT to science, engineering and technology and highlights public outreach and direct educational benefits. South Africa, more so than other 
developing countries, requires a sustained campaign to promote at all levels of society an awareness and understanding of SET and their importance in the empowerment of individuals and communities to ensure the international competitiveness of the nation. Until recently black people in South Africa had very limited access to SET education, training and careers. The specific objective of the public outreach programme is the enhancement of science education and awareness throughout South African society. Students and citizens of South Africa will be inspired and encouraged by the accomplishments of SALT (National Research Foundation 1999).

The plan identifies several aspects of the SEI that it will support, in particular resource development, teacher training in these resources and the 'Starbus' project. In addition it envisages several public awareness programmes including:

- an Internet site showing SALT under construction

- an educational video on SALT

- the creation of SALT models and posters for display at exhibitions and other key public areas such as planetaria, museums and science festivals

- a daily two-minute radio slot, a southern hemisphere version of the Stardate programme, in collaboration with McDonald Observatory.

\section{Acknowledgments}

I would like to thank Drs Patricia Whitelock and Peter Martinez for their support with the SEI and Susan Heese for her suggestions and contributions to this paper. I would also like to thank Professor Julietta Fierro for inviting me to attend the Astronomy Education in the New Millennium conference, and the IAU and SAAO for funding.

\section{References}

Department of Arts, Culture, Science and Technology 1996 , White Paper on Science and Technology, Preparing for the 21st Century

Department of Education 1997, Curriculum 2005-A Discussion Document

Eames, C., \& Eames, R. 1989, Powers of Ten (Santa Monica, CA: Pyramid Home Video)

Enderstein, L. G., \& Spargo, P. E. 1998, The effect of context, culture and learning on the selection of alternative options in similar situations by South African pupils. Int. J. Sci. Educ., 20, 711-36

Heese, S. 1998, Taking science to the people. Proc. 2nd National Conf. on Public Understanding of Science and Technology in South Africa, Pretoria

IAU Commission 46 1999, The Teaching of Astronomy Newsletter 50

National Research Foundation 1999, SALT Collateral Benefits Plan

Prigogine, I. 1980, From Being to Becoming (San Francisco: Freeman)

Project STAR 1989, A Private Universe (Santa Monica, CA: Pyramid Home Video)

Rijsdijk, C. L. 1995, Looking for links. Proc. 16th National Convention of the Federation of Natural Science and Mathematics of South Africa

Rijsdijk, C. L. 1996, SAAO internal policy document

Rijsdijk, C. L. 1998, SAAO business plan for the 'Friends with the Universe' project

Stone, R. 1998, Outcomes assessment—workshop for teachers at the Western Cape College of Education (unpublished) 